\title{
Effect of Diabetes and Admission Blood Glucose Levels on Short-term Outcomes in Patients with Critical illnesses: A Real-world Study Based on Propensity Score Analyses
}

Shan Lin

Sun Yat-sen University First Affiliated Hospital

Shanhui Ge

Sun Yat-sen University First Affiliated Hospital

Wanmei He

Sun Yat-sen University First Affiliated Hospital

Mian Zeng ( $\square$ zengmian@mail.sysu.edu.cn )

Sun Yat-sen University First Affiliated Hospital

Research

Keywords: critical care, diabetes, glucose, prognosis

Posted Date: September 30th, 2020

DOl: https://doi.org/10.21203/rs.3.rs-55105/v1

License: (c) (i) This work is licensed under a Creative Commons Attribution 4.0 International License.

Read Full License 


\section{Abstract}

Background: Effect of diabetes and optimal range of blood glucose level on the short-term prognosis in patients with critical illnesses are currently ambiguous. We aimed to determine whether diabetes affects short-term prognosis and optimal range of blood glucose level for critically ill patients.

Methods: We performed a retrospective analysis of data on 46,476 critically ill patients from the critical care database (version 1.4), collected prospectively between 2002 and 2012. Association of diabetes with 28-day mortality was assessed by inverse probability weighting based on the propensity score. Smoothing splines and threshold effect analysis were applied to explore the optimal glucose range.

Results: Of the 33,680 patients enrolled in the study, 8,701 (25.83\%) had diabetes. In the main analysis, the 28 -day mortality was reduced by $29 \%$ (hazard ratio $(\mathrm{HR})=0.71,95 \%$ confidence interval $(\mathrm{Cl}) 0.67-0.76$ ) in patients with diabetes compared to those without diabetes. The E-value of 2.17 indicated robustness to unmeasured confounders. The effect of the association between with diabetes and 28-day mortality was generally in line for all subgroup variables, significant interactions were observed for glucose at ICU admission, admission type, and insulin use (Interaction $P<0.05$ ). A V-shaped relationship was observed between glucose and 28-day mortality in patients without diabetes, with the lowest 28-day mortality corresponding to the glucose level was $101.75 \mathrm{mg} / \mathrm{dl}$ (95\% Cl 94.64-105.80 mg/dl), and hypoglycemia or hyperglycemia should be avoided, especially in patients admitted to the surgical intensive care unit (SICU), cardiac surgery recovery unit (CSRU), and coronary care unit (CCU); for patients with diabetes, no optimal threshold for glucose was found, and elevated blood glucose does not appear to be associated with a poor prognosis, and perhaps may be beneficial except for CCU and CSRU.

\section{Conclusions:}

The non-detrimental effect of diabetes on the short-term prognosis of critically ill patients was further confirmed, which would reduce 28 -day mortality by approximately $29 \%$. For patients without diabetes, the glucose level corresponding to the lowest 28-day mortality was 101.75 mg/dl (95\% Cl 94.64-105.80 $\mathrm{mg} / \mathrm{dl})$; however, for patients with diabetes, the appropriate optimal blood glucose threshold remains unresolved.

\section{Background}

Diabetes is rapidly emerging as a pandemic worldwide. Of the overall U.S. population, approximately 34.2 million people had developed diabetes in 2018 , of which 34.1 million were adults (> 18 years), accounting for $13.0 \%$ of all U.S. adults [1]. In critical ill patients admitted to the intensive care units (ICUs), diabetes is a relatively frequent diagnosis. Diabetes occasionally leads to ICU admissions but is usually a part of a comorbid condition. As diabetes is a multifaceted disease that can lead to immune system impairments and metabolic dysregulations, treatment of patients is complicated, potentially increasing the severity of the primary disease $[2,3]$. On reviewing studies over the past 20 years, the percentage of critically ill patients with diabetes as a comorbid condition was found to range from $13.2 \%$ to $30.4 \%$ [4-14]. 
Furthermore, these studies were inconclusive about the relationship between diabetes and the prognosis in patients with acute illness. Some studies suggested that in critically ill patients, diabetes increased the risk of mortality as well as the incidence of infection [8-10]. Other studies suggested that diabetes may not negatively affect the clinical outcomes of critically ill patients and may even be protective, which seems to conflict with clinical judgment [11-13].

In addition, there is no consensus on the scope of optimal glycemic control to date. In the past two decades, numerous clinical trials have attempted to explore the optimal range of glycemic control to reduce mortality in critically ill patients; however, these clinical trials have yielded conflicting results [4-7, 14-19]. From the first clinical trial in 2001 until now, there has only been a consensus that the safest range for glycemic control in critically ill patients should not be higher than $180 \mathrm{mg} / \mathrm{dl}$; however, the safest range for glycemic control is still unknown [4]. In addition, the quality of glycemic control in critically ill patients has been suggested to be closely related to prognosis [20]. Indeed, poor glycemic control may result in glycemic variability, either hypoglycemia or hyperglycemia, which could be associated with negative outcomes in patients with critical illness. Determining the optimal range of glycemic control from the results of clinical trials with large sample sizes is difficult, and previous clinical trials have been both hard-won and praiseworthy. Considering the susceptibility to variations in blood glucose levels in critically ill patients after ICU admission, we tested blood glucose levels at admission to retrospectively investigate whether there is an optimal blood glucose level range for critically ill patients and, if so, to determine this range.

The objectives of our study were to determine 1) whether diabetes affects short-term prognosis and 2) the optimal range of blood glucose level in critically ill patients.

\section{Methods}

\section{Patient data}

We carried out a retrospective analysis of data on 46,476 critically ill patients from the Critical Care Multiparameter Database III (MIMIC-III) version 1.4, collected prospectively between 2002 and 2012 [21]. The Beth Israel Deaconess Medical Center and the institutional review boards of MIT-affiliated institutions granted permission to access the database (Record ID: 33460949). The requirement for patient consent was waived because of the anonymity of data.

All adult ( $\geq 18$ years) patients were recruited for the study, but patients with a follow-up of $>1$ day were included in the analysis. We only analyzed patients who were admitted to the ICU for the first time. Blood glucose level was determined on the basis of the first plasma glucose level obtained in the patients admitted to the ICUs, which was measured by hospital laboratory staff and uploaded onto the electronic medical records system. Together with conventional variables such as demographic characteristics (age, sex, admission type, etc.), therapeutic interventions, and clinical outcomes, we simultaneously extracted data on the simplified acute physiology score II (SAPS II) [22], sepsis, and specified comorbidities. 
Therapeutic interventions included the requirement for mechanical ventilation (MV) or renal replacement therapy (RRT) on the first day and insulin for the entire ICU period. Data were extracted using Structured Query Language (SQL), and the code to support MIMIC-III documentation and websites is publicly available (https://github.com/MIT-LCP/mimic-code) [23, 24].

\section{Outcomes}

The primary outcomes were 28-day mortality in critically ill patients with diabetes and the optimal blood glucose level range.

\section{Statistical analysis}

Data were expressed as mean \pm standard deviation or median (interquartile range, IQR) for continuous variables, with comparison of group characteristics using the Kruskal-Wallis test (or Fisher's exact test); categorical variables were expressed as numbers and percentages, which were compared using the Chisquare test. We constructed propensity score methods utilizing the following three propensity score models: covariate-adjusted propensity scores, propensity score matching (PSM), and inverse probability weighting based on the propensity score. A logistic regression model was used to compute the propensity scores, which consisted of the following baseline covariates: age, sex, sepsis, admission type, use of insulin, type of ICU at admission, SAPS II, MV on the first day, blood glucose level at ICU admission, RRT on the first day, cardiac arrhythmias, congestive heart failure, peripheral vascular disease, valvular disease, hypertension, other neurological diseases, chronic pulmonary disease, renal failure, liver disease, acquired immune deficiency syndrome (AIDS), lymphoma, metastatic cancer, solid tumor, obesity, fluid and electrolyte disorders, alcohol abuse, drug abuse, and depression [25]. Propensity score matching was performed using a 1:1 matching protocol without replacement (greedy-matching algorithm), with a caliper width equal to 0.05 of the standard deviation of the logit of the propensity score. For the inverseprobability-weighted analysis, we used the predicted probability of a propensity score model to obtain stable inverse probability weights [26]. Standardized differences between covariates that were less than $10.0 \%$ represented a relatively balanced condition [27]. The adjustment variables in the multivariate analysis model were consistent with those in the propensity score models. The results of the main analysis were used along with inverse probability weighting. We also calculated E-values to explore the potential effect of unmeasured confounders of diabetes and 28-day mortality [28, 29]. The E-value allowed for quantifying the required magnitude of an unmeasured confounding factor that might counteract the observed correlation between diabetes and 28-day mortality. The Kaplan-Meier method (log-rank test) was used to describe survival differences of the PSM cohort.

Subsequently, to determine whether there was a U-shaped association between 28-day mortality and blood glucose levels on admission, we performed a smoothing splines using a Cox model to fit the 28day mortality. Inflection points were measured by the two-piece-wise regression model constructed in the threshold effects analysis, with the differences compared via the log-likelihood ratio test and the $95 \%$ 
confidence interval $(\mathrm{Cl})$ for the inflection point computed using the bootstrap method [30]. Finally, we performed stratified analyses and interaction tests to explore the concordance of the associations for outcomes within different subgroup variables, which included demographic characteristics (e.g., age, sex, etc.), therapeutic regimens (e.g., use of insulin or not), different glucose concentrations, types of ICUs, sepsis, and SAPS II. EmpowerStats (www.empowerstats.com) and R (http://www.R-project.org, version 3.4.3) were applied to all data analyses. A $P$ value $<0.05$ was considered statistically significant.

\section{Results}

\section{Characteristics of the original cohort}

In this study, 33,680 patients with a mean age of 63.65 years were enrolled, among whom 8,701 had diabetes, accounting for about $25.83 \%$ of patients in the original cohort [Table 1 and Figure 1]. Before propensity score matching, there were several differences in the baseline variables between the groups of patients with and without diabetes. With propensity score matching in a 1:1 ratio, 7,261 patients with diabetes were matched with 7,261 patients without diabetes. The model had a C-statistic of 0.8214 . After matching, all variables had standardized differences less than $10.0 \%$, which suggested that the betweengroup difference was relatively small [Table 2]. 
Table 1

Characteristics of original cohort

\begin{tabular}{|c|c|c|c|c|}
\hline Variables & $\begin{array}{l}\text { All patients } \\
(\mathrm{N}=33680)\end{array}$ & $\begin{array}{l}\text { Without diabetes } \\
(\mathrm{N}=24979)\end{array}$ & $\begin{array}{l}\text { With diabetes } \\
(\mathrm{N}=8701)\end{array}$ & $\begin{array}{l}\text { Standardized } \\
\text { Difference, \% }\end{array}$ \\
\hline Age (years) & $63.65 \pm 17.40$ & $62.44 \pm 18.28$ & $67.10 \pm 14.02$ & 28.6 \\
\hline Sex & & & & 2.4 \\
\hline Male & $\begin{array}{l}19103 \\
(56.72 \%)\end{array}$ & 14090 (56.41\%) & $5013(57.61 \%)$ & \\
\hline Female & $\begin{array}{l}14577 \\
(43.28 \%)\end{array}$ & 10889 (43.59\%) & 3688 (42.39\%) & \\
\hline Admission type & & & & 3.3 \\
\hline Emergency & $\begin{array}{l}27691 \\
(82.22 \%)\end{array}$ & 20502 (82.08\%) & $7189(82.62 \%)$ & \\
\hline Elective & $\begin{array}{l}5426 \\
(16.11 \%)\end{array}$ & $4079(16.33 \%)$ & 1347 (15.48\%) & \\
\hline Urgent & $563(1.67 \%)$ & $398(1.59 \%)$ & $165(1.90 \%)$ & \\
\hline $\begin{array}{l}\text { Type of ICU on } \\
\text { admission }\end{array}$ & & & & 26.3 \\
\hline $\mathrm{CCU}$ & $\begin{array}{l}4811 \\
(14.28 \%)\end{array}$ & $3344(13.39 \%)$ & $1467(16.86 \%)$ & \\
\hline CSRU & $\begin{array}{l}6349 \\
(18.85 \%)\end{array}$ & $4434(17.75 \%)$ & $1915(22.01 \%)$ & \\
\hline MICU & $\begin{array}{l}12071 \\
(35.84 \%)\end{array}$ & 8811 (35.27\%) & $3260(37.47 \%)$ & \\
\hline SICU & $\begin{array}{l}5623 \\
(16.70 \%)\end{array}$ & $4320(17.29 \%)$ & 1303 (14.98\%) & \\
\hline TSICU & $\begin{array}{l}4826 \\
(14.33 \%)\end{array}$ & 4070 (16.29\%) & $756(8.69 \%)$ & \\
\hline $\begin{array}{l}\text { Glucose at ICU } \\
\text { admission }(\mathrm{mg} / \mathrm{dl})\end{array}$ & $137.20 \pm 40.96$ & $128.39 \pm 32.52$ & $162.15 \pm 50.92$ & 79.0 \\
\hline Sepsis & $\begin{array}{l}11265 \\
(33.45 \%)\end{array}$ & 8037 (32.18\%) & 3228 (37.10\%) & 10.4 \\
\hline Use of insulin & $\begin{array}{l}19061 \\
(56.59 \%)\end{array}$ & $12092(48.41 \%)$ & 6969 (80.09\%) & 70.1 \\
\hline SAPS II & $\begin{array}{l}33.00(24.00- \\
42.00)\end{array}$ & $\begin{array}{l}32.00(23.00- \\
41.00)\end{array}$ & $\begin{array}{l}35.00(27.00- \\
44.00)\end{array}$ & 22.5 \\
\hline MV on the first day & $\begin{array}{l}15262 \\
(45.31 \%)\end{array}$ & 11260 (45.08\%) & 4002 (45.99\%) & 1.8 \\
\hline
\end{tabular}




\begin{tabular}{|c|c|c|c|c|}
\hline RRT on the first day & $922(2.74 \%)$ & $513(2.05 \%)$ & $409(4.70 \%)$ & 14.7 \\
\hline \multicolumn{5}{|l|}{ Other comorbidities } \\
\hline $\begin{array}{l}\text { Congestive heart } \\
\text { failure }\end{array}$ & $\begin{array}{l}8141 \\
(24.17 \%)\end{array}$ & $5192(20.79 \%)$ & 2949 (33.89\%) & 29.7 \\
\hline Cardiac arrhythmias & $\begin{array}{l}9785 \\
(29.05 \%)\end{array}$ & $6910(27.66 \%)$ & $2875(33.04 \%)$ & 11.7 \\
\hline Valvular disease & $\begin{array}{l}5267 \\
(15.64 \%)\end{array}$ & 3882 (15.54\%) & $1385(15.92 \%)$ & 1.0 \\
\hline $\begin{array}{l}\text { Peripheral vascular } \\
\text { disease }\end{array}$ & $\begin{array}{l}3478 \\
(10.33 \%)\end{array}$ & 2307 (9.24\%) & 1171 (13.46\%) & 13.3 \\
\hline Hypertension & $\begin{array}{l}18544 \\
(55.06 \%)\end{array}$ & 12153 (48.65\%) & 6391 (73.45\%) & 52.6 \\
\hline $\begin{array}{l}\text { Other neurological } \\
\text { disease }\end{array}$ & $\begin{array}{l}4124 \\
(12.24 \%)\end{array}$ & 3246 (12.99\%) & 878 (10.09\%) & 9.1 \\
\hline $\begin{array}{l}\text { Chronic pulmonary } \\
\text { disease }\end{array}$ & $\begin{array}{l}6251 \\
(18.56 \%)\end{array}$ & 4499 (18.01\%) & $1752(20.14 \%)$ & 5.4 \\
\hline Liver disease & 2407 (7.15\%) & 1709 (6.84\%) & $698(8.02 \%)$ & 4.5 \\
\hline Renal failure & $\begin{array}{l}4254 \\
(12.63 \%)\end{array}$ & 2225 (8.91\%) & 2029 (23.32\%) & 40.0 \\
\hline AIDS & $303(0.90 \%)$ & $263(1.05 \%)$ & $40(0.46 \%)$ & 6.9 \\
\hline Lymphoma & $583(1.73 \%)$ & $465(1.86 \%)$ & 118 (1.36\%) & 4.0 \\
\hline Metastatic cancer & 2137 (6.35\%) & $1764(7.06 \%)$ & 373 (4.29\%) & 12.0 \\
\hline Solid tumor & $1717(5.10 \%)$ & $1294(5.18 \%)$ & $423(4.86 \%)$ & 1.5 \\
\hline Obesity & $1865(5.54 \%)$ & $902(3.61 \%)$ & $963(11.07 \%)$ & 28.9 \\
\hline $\begin{array}{l}\text { Fluid and electrolyte } \\
\text { disorders }\end{array}$ & $\begin{array}{l}9573 \\
(28.42 \%)\end{array}$ & $6820(27.30 \%)$ & 2753 (31.64\%) & 9.5 \\
\hline Alcohol abuse & $2812(8.35 \%)$ & $2418(9.68 \%)$ & $394(4.53 \%)$ & 20.2 \\
\hline Drug abuse & $1217(3.61 \%)$ & $1069(4.28 \%)$ & $148(1.70 \%)$ & 15.2 \\
\hline Depression & $2878(8.55 \%)$ & $2124(8.50 \%)$ & 754 (8.67\%) & 0.6 \\
\hline \multicolumn{5}{|c|}{$\begin{array}{l}\text { Abbreviations: ICU: intensive care unit; SAPS II: simplified acute physiology score II; AIDS: acquired } \\
\text { immune deficiency syndrome; CCU: coronary care unit; CSRU: cardiac surgery recovery unit; MICU: } \\
\text { medical intensive care unit; SICU: surgical intensive care unit; TSICU: trauma/surgical intensive care } \\
\text { unit; MV: mechanical ventilation; RRT: renal replacement therapy. }\end{array}$} \\
\hline
\end{tabular}


Table 2

Characteristics of PSM cohort

\begin{tabular}{|c|c|c|c|c|}
\hline Variables & $\begin{array}{l}\text { All patients } \\
(\mathrm{N}=14,522)\end{array}$ & $\begin{array}{l}\text { Without diabetes } \\
(\mathrm{N}=7,261)\end{array}$ & $\begin{array}{l}\text { With diabetes } \\
(\mathrm{N}=7,261)\end{array}$ & $\begin{array}{l}\text { Standardized } \\
\text { Difference, \% }\end{array}$ \\
\hline Age (years) & $67.47 \pm 14.73$ & $67.84 \pm 15.32$ & $67.10 \pm 14.11$ & 5.0 \\
\hline Gender & & & & 0.8 \\
\hline Male & 8404 (57.87\%) & 4217 (58.08\%) & $4187(57.66 \%)$ & \\
\hline Female & $6118(42.13 \%)$ & 3044 (41.92\%) & 3074 (42.34\%) & \\
\hline Admission type & & & & 4.4 \\
\hline Emergency & $\begin{array}{l}11639 \\
(80.15 \%)\end{array}$ & 5757 (79.29\%) & $5882(81.01 \%)$ & \\
\hline Elective & $2614(18.00 \%)$ & $1368(18.84 \%)$ & $1246(17.16 \%)$ & \\
\hline Urgent & $269(1.85 \%)$ & $136(1.87 \%)$ & $133(1.83 \%)$ & \\
\hline $\begin{array}{l}\text { Type of ICU on } \\
\text { admission }\end{array}$ & & & & 9.5 \\
\hline $\mathrm{CCU}$ & $2273(15.65 \%)$ & $1194(16.44 \%)$ & 1079 (14.86\%) & \\
\hline CSRU & 3707 (25.53\%) & $1908(26.28 \%)$ & $1799(24.78 \%)$ & \\
\hline MICU & 4747 (32.69\%) & $2180(30.02 \%)$ & 2567 (35.35\%) & \\
\hline SICU & $2348(16.17 \%)$ & $1220(16.80 \%)$ & $1128(15.54 \%)$ & \\
\hline TSICU & 1447 (9.96\%) & 759 (10.45\%) & $688(9.48 \%)$ & \\
\hline $\begin{array}{l}\text { Glucose at ICU } \\
\text { admission (mg/dl) }\end{array}$ & 149.19 (41.03) & $147.32(41.61)$ & $151.07(40.35)$ & 9.1 \\
\hline Sepsis & 5667 (39.02\%) & $2883(39.71 \%)$ & $2784(38.34 \%)$ & 2.8 \\
\hline Use of insulin & $\begin{array}{l}11207 \\
(77.17 \%)\end{array}$ & $5613(77.30 \%)$ & $5594(77.04 \%)$ & 0.6 \\
\hline SAPS II & $\begin{array}{l}35.00(27.00- \\
44.00)\end{array}$ & $\begin{array}{l}35.00(27.00- \\
44.00)\end{array}$ & $\begin{array}{l}35.00(27.00- \\
44.00)\end{array}$ & 2.0 \\
\hline MV on the first day & 7245 (49.89\%) & $3689(50.81 \%)$ & 3556 (48.97\%) & 3.7 \\
\hline RRT on the first day & $581(4.00 \%)$ & 289 (3.98\%) & $292(4.02 \%)$ & 0.2 \\
\hline \multicolumn{5}{|l|}{ Other comorbidities } \\
\hline $\begin{array}{l}\text { Congestive heart } \\
\text { failure }\end{array}$ & $4566(31.44 \%)$ & $2274(31.32 \%)$ & 2292 (31.57\%) & 0.5 \\
\hline
\end{tabular}




\begin{tabular}{|c|c|c|c|c|}
\hline Cardiac arrhythmias & $4978(34.28 \%)$ & $2534(34.90 \%)$ & $2444(33.66 \%)$ & 2.6 \\
\hline Valvular disease & $2540(17.49 \%)$ & $1301(17.92 \%)$ & 1239 (17.06\%) & 2.2 \\
\hline $\begin{array}{l}\text { Peripheral vascular } \\
\text { disease }\end{array}$ & 1993 (13.72\%) & $1033(14.23 \%)$ & $960(13.22 \%)$ & 2.9 \\
\hline Hypertension & $\begin{array}{l}10319 \\
(71.06 \%)\end{array}$ & $5180(71.34 \%)$ & 5139 (70.78\%) & 1.2 \\
\hline $\begin{array}{l}\text { Other neurological } \\
\text { disease }\end{array}$ & $1492(10.27 \%)$ & 733 (10.10\%) & 759 (10.45\%) & 1.2 \\
\hline $\begin{array}{l}\text { Chronic pulmonary } \\
\text { disease }\end{array}$ & 2898 (19.96\%) & 1447 (19.93\%) & 1451 (19.98\%) & 0.1 \\
\hline Liver disease & 1070 (7.37\%) & $536(7.38 \%)$ & $534(7.35 \%)$ & 0.1 \\
\hline Renal failure & 2758 (18.99\%) & $1346(18.54 \%)$ & 1412 (19.45\%) & 2.3 \\
\hline AIDS & $60(0.41 \%)$ & $28(0.39 \%)$ & $32(0.44 \%)$ & 0.9 \\
\hline Lymphoma & $233(1.60 \%)$ & $122(1.68 \%)$ & $111(1.53 \%)$ & 1.2 \\
\hline Metastatic cancer & $688(4.74 \%)$ & 345 (4.75\%) & $343(4.72 \%)$ & 0.1 \\
\hline Solid tumor & $742(5.11 \%)$ & $375(5.16 \%)$ & 367 (5.05\%) & 0.5 \\
\hline Obesity & $1224(8.43 \%)$ & $586(8.07 \%)$ & $638(8.79 \%)$ & 2.6 \\
\hline $\begin{array}{l}\text { Fluid and electrolyte } \\
\text { disorders }\end{array}$ & $4305(29.64 \%)$ & $2156(29.69 \%)$ & $2149(29.60 \%)$ & 0.2 \\
\hline Alcohol abuse & $690(4.75 \%)$ & $329(4.53 \%)$ & 361 (4.97\%) & 2.1 \\
\hline Drug abuse & $260(1.79 \%)$ & $127(1.75 \%)$ & $133(1.83 \%)$ & 0.6 \\
\hline Depression & 1163 (8.01\%) & $567(7.81 \%)$ & $596(8.21 \%)$ & 1.5 \\
\hline \multicolumn{5}{|c|}{$\begin{array}{l}\text { Abbreviations: PSM: propensity score matching; ICU: intensive care unit; SAPS II: simplified acute } \\
\text { physiology score II; AIDS: acquired immune deficiency syndrome; CCU: coronary care unit; CSRU: } \\
\text { cardiac surgery recovery unit; MICU: medical intensive care unit; SICU: surgical intensive care unit; } \\
\text { TSICU: trauma/surgical intensive care unit; MV: mechanical ventilation; RRT: renal replacement } \\
\text { therapy. }\end{array}$} \\
\hline
\end{tabular}

\section{Clinical outcomes in the original cohort and PSM cohort}

Altogether, in the original cohort, there were no obvious differences in the 28-day mortality, in-hospital mortality, or ICU mortality between the two groups (all $P>0.05$ ) [Table 3]. After matching, patients with diabetes had a significantly lower 28-day, ICU, and in-hospital mortality than did those without diabetes (all $P<0.001$ ); Kaplan-Meier curves indicated that patients with diabetes had a considerably better survival advantage than did matched patients without diabetes (log-rank test: $P<0.0001$ ) [Figure 2]. 
Table 3

Clinical outcomes in the original cohort and the PSM cohort

\begin{tabular}{|c|c|c|c|c|c|c|}
\hline & Original cohort & & & PSM cohort & & \\
\hline $\begin{array}{l}\text { Clinical } \\
\text { Outcomes }\end{array}$ & $\begin{array}{l}\text { Without } \\
\text { diabetes } \\
(\mathrm{N}=24979)\end{array}$ & $\begin{array}{l}\text { With } \\
\text { diabetes } \\
(\mathrm{N}=8701)\end{array}$ & $\begin{array}{l}P \text { - } \\
\text { value }\end{array}$ & $\begin{array}{l}\text { Without } \\
\text { diabetes } \\
(\mathrm{N}=7,261)\end{array}$ & $\begin{array}{l}\text { With } \\
\text { diabetes } \\
(\mathrm{N}=7,261)\end{array}$ & $\begin{array}{l}P \text { - } \\
\text { value }\end{array}$ \\
\hline $\begin{array}{l}\text { 28-day } \\
\text { mortality, } \\
\text { n(\%) }\end{array}$ & 2715 (10.87\%) & $\begin{array}{l}957 \\
(11.00 \%)\end{array}$ & 0.738 & $964(13.28 \%)$ & $\begin{array}{l}798 \\
(10.99 \%)\end{array}$ & $<0.001$ \\
\hline $\begin{array}{l}\text { ICU } \\
\text { mortality, } \\
\mathrm{n}(\%)\end{array}$ & 1502 (6.01\%) & $493(5.67 \%)$ & 0.238 & $588(8.10 \%)$ & 403 (5.55\%) & $<0.001$ \\
\hline $\begin{array}{l}\text { Hospital } \\
\text { mortality, } \\
\mathrm{n}(\%)\end{array}$ & 2300 (9.21\%) & 769 (8.84\%) & 0.302 & 855 (11.78\%) & $626(8.62 \%)$ & $<0.001$ \\
\hline
\end{tabular}

\section{Associations between with diabetes and clinical outcomes}

As shown in Table 4, a multivariable model with inverse probability weighting based on the propensity score showed that the 28 -day mortality rate was reduced by $29 \%$ (hazard ratio $(\mathrm{HR})=0.71,95 \% \mathrm{Cl} 0.67$ $0.76)$ in the group with diabetes compared with the group without diabetes. The estimated E-value was 2.17 (upper confidence limit 1.96), which means that if there were no immeasurable confounders associated with diabetes as well as 28 -day mortality, with relative risks of $\geq 2.17$ for both, then the results we obtained were robust. 
Table 4

Associations between diabetes and clinical outcomes in the crude analysis, multivariable Cox analysis, and propensity score analyses

\begin{tabular}{|c|c|c|c|}
\hline \multicolumn{4}{|l|}{ Clinical outcomes } \\
\hline 28-day mortality & Groups & $\mathrm{HR}(95 \% \mathrm{Cl})$ & $P$-value \\
\hline \multirow[t]{2}{*}{ Crude analysis } & Without diabetes & Ref. & - \\
\hline & With diabetes & $1.01(0.94-1.09)$ & 0.8045 \\
\hline \multirow[t]{2}{*}{ Multivariable analysis } & Without diabetes & Ref. & - \\
\hline & With diabetes & $0.86(0.79-0.93)$ & 0.0004 \\
\hline \multicolumn{4}{|l|}{ Propensity-score models } \\
\hline \multirow[t]{2}{*}{ Adjusted for propensity score } & Without diabetes & Ref. & - \\
\hline & With diabetes & $0.90(0.83-0.98)$ & 0.0132 \\
\hline \multirow[t]{2}{*}{ With matching } & Without diabetes & Ref. & - \\
\hline & With diabetes & $0.81(0.74-0.89)$ & $<0.0001$ \\
\hline \multirow[t]{2}{*}{ With inverse probability weighting } & Without diabetes & Ref. & - \\
\hline & With diabetes & $0.71(0.67-0.76)$ & $<0.0001$ \\
\hline \multicolumn{4}{|c|}{$\begin{array}{l}\text { Multivariable analysis adjusted for age, sex, sepsis, admission type, use of insulin, type of ICU on } \\
\text { admission, SAPS II, glucose at ICU admission, MV on the first day, RRT on the first day, cardiac } \\
\text { arrhythmias, congestive heart failure, peripheral vascular disease, valvular disease, hypertension, } \\
\text { other neurological diseases, chronic pulmonary disease, renal failure, liver disease, AIDS, lymphoma, } \\
\text { metastatic cancer, solid tumor, obesity, fluid and electrolyte disorders, alcohol abuse, drug abuse, and } \\
\text { depression. }\end{array}$} \\
\hline \multicolumn{4}{|c|}{$\begin{array}{l}\text { Propensity score was calculated by age, sex, sepsis, admission type, use of insulin, type of ICU on } \\
\text { admission, SAPS II, glucose at ICU admission, MV on the first day, RRT on the first day, cardiac } \\
\text { arrhythmias, congestive heart failure, peripheral vascular disease, valvular disease, hypertension, } \\
\text { other neurological diseases, chronic pulmonary disease, renal failure, liver disease, AIDS, lymphoma, } \\
\text { metastatic cancer, solid tumor, obesity, fluid and electrolyte disorders, alcohol abuse, drug abuse, and } \\
\text { depression. }\end{array}$} \\
\hline \multicolumn{4}{|c|}{$\begin{array}{l}\text { Propensity score matching was performed with the use of a } 1: 1 \text { matching protocol without } \\
\text { replacement (greedy-matching algorithm), with a caliper width equal to } 0.05 \text { of the standard deviation } \\
\text { of the logit of the propensity score. }\end{array}$} \\
\hline \multicolumn{4}{|c|}{ Inverse probability weighting was used with the same covariates according to the propensity score. } \\
\hline \multicolumn{4}{|c|}{ Abbreviations: HR, hazard ratio; $\mathrm{Cl}$, confidence interval. } \\
\hline
\end{tabular}

\section{Stratified analyses and interaction tests}


Altogether, the effect of the association between diabetes and 28-day mortality was generally in line for all subgroup variables [Table 5]. Significant interactions were observed for blood glucose levels at ICU admission, admission type, and insulin use (Interaction $P<0.05$ ). Glucose levels of $140-200 \mathrm{mg} / \mathrm{dl}$ and $\geq 200 \mathrm{mg} / \mathrm{dl}$ were associated with a remarkable reduction in 28-day mortality in patients with diabetes as compared with those without diabetes $(\mathrm{HR}=0.84,95 \% \mathrm{Cl} 0.74-0.95$ and $\mathrm{HR}=0.49,95 \% \mathrm{Cl}$ 0.46-0.70, respectively). Patients with diabetes admitted as emergency and urgent cases were differentially decreased $(\mathrm{HR}=0.85,95 \% \mathrm{Cl} 0.78-0.93$ and $\mathrm{HR}=0.34,95 \% \mathrm{Cl}$ 0.15-0.77, respectively); however, no significant reduction in 28-day mortality was observed among patients admitted elective. On treatment with insulin, the 28-day mortality was markedly reduced in patients with diabetes ( $\mathrm{HR}=0.76,95 \% \mathrm{Cl} 0.68$ 0.84), whereas a corresponding beneficial effect in patients with diabetes in whom insulin was not used was not attained $(\mathrm{HR}=1.09,95 \% \mathrm{Cl} 0.93-1.27)$. 
Table 5

Effect size of diabetes on 28-day mortality rate in prespecified and exploratory subgroups in each subgroup

\begin{tabular}{|c|c|c|c|c|}
\hline$Y=28-$ day mortality & Adjusted & & & \\
\hline & $\begin{array}{l}\text { Without } \\
\text { diabetes }\end{array}$ & $\begin{array}{l}\text { With diabetes, HR (95\% } \\
\mathrm{Cl})\end{array}$ & $P$-value & $\begin{array}{l}P \text { for } \\
\text { interaction }\end{array}$ \\
\hline Age (years) & & & & 0.3461 \\
\hline$<65$ & 1.0 & $0.86(0.72-1.01)$ & 0.0732 & \\
\hline$\geq 65$ & 1.0 & $0.86(0.78-0.95)$ & 0.0021 & \\
\hline Sex & & & & 0.7880 \\
\hline Male & 1.0 & $0.85(0.76-0.96)$ & 0.0067 & \\
\hline Female & 1.0 & $0.87(0.77-0.98)$ & 0.0242 & \\
\hline $\begin{array}{l}\text { Glucose at ICU admission } \\
(\mathrm{mg} / \mathrm{dl})\end{array}$ & & & & $<0.0001$ \\
\hline$<140$ & 1.0 & $1.03(0.91-1.17)$ & 0.6588 & \\
\hline$\geq 140,<200$ & 1.0 & $0.84(0.74-0.95)$ & 0.0061 & \\
\hline$\geq 200$ & 1.0 & $0.57(0.46-0.70)$ & $<0.0001$ & \\
\hline Admission type & & & & 0.0280 \\
\hline Emergency & 1.0 & $0.85(0.78-0.93)$ & 0.0003 & \\
\hline Elective & 1.0 & $1.22(0.80-1.84)$ & 0.3529 & \\
\hline Urgent & 1.0 & $0.34(0.15-0.77)$ & 0.0101 & \\
\hline Type of ICU on admission & & & & 0.1173 \\
\hline $\mathrm{CCU}$ & 1.0 & $0.61(0.49-0.76)$ & $<0.0001$ & \\
\hline CSRU & 1.0 & $0.94(0.66-1.33)$ & 0.7151 & \\
\hline MICU & 1.0 & $0.94(0.83-1.06)$ & 0.3221 & \\
\hline SICU & 1.0 & $0.81(0.66-0.98)$ & 0.0315 & \\
\hline TSICU & 1.0 & $0.96(0.74-1.23)$ & 0.7236 & \\
\hline SAPS II & & & & 0.1625 \\
\hline$<30$ & 1.0 & $0.69(0.50-0.94)$ & 0.0202 & \\
\hline$\geq 30,<60$ & 1.0 & $0.79(0.71-0.87)$ & $<0.0001$ & \\
\hline
\end{tabular}


significant, namely, the HR for glucose $<101.75$ was 0.98 , and the HR for glucose $\geq 101.75$ was 1.01 ( $P$ value for log-likelihood ratio test, $<0.001$ ) [Table 5]. As for the 28-day mortality in patients with diabetes, threshold effects analysis revealed that no significant differences were observed in either the one-line linear regression model or the two-piece-wise linear regression model ( $P$-value for log-likelihood ratio test, 0.132) [Table 5]. Additionally, we fitted smoothing splines for the relationship between glucose and 28day mortality in diabetic and non-diabetic patients in different ICUs and further analyzed the effects of different glucose level groups on 28-day mortality in diabetic and non-diabetic patients in different ICUs [Table 7, and Figure 4 and 5]. As shown in Table 7, we found that in non-diabetic patients, blood glucose level $>200 \mathrm{mg} / \mathrm{dl}$ had variable adverse effects on different ICU patients, especially those in the cardiac surgery recovery unit (CSRU) (HR=3.68, 95\% Cl 1.57-8.60). Moreover, a blood glucose level $<70 \mathrm{mg} / \mathrm{dl}$, especially among patients in the surgical intensive care unit (SICU), was associated with a significant increase in 28-day mortality ( $\mathrm{HR}=10.17,95 \% \mathrm{Cl} 2.49-41.57)$. In diabetic patients, hyperglycemia $(\geq 200$ $\mathrm{mg} / \mathrm{dl}$ ) was equally detrimental to the patients in the coronary care unit (CCU) and those in the CSRU $(\mathrm{HR}=2.35,95 \% \mathrm{Cl} 1.44-3.81$ and $\mathrm{HR}=3.53,95 \% \mathrm{Cl} 1.37-9.12)$, whereas in the other ICUs, hyperglycemia was not associated with a poor prognosis, and is perhaps beneficial for the patients. 
Table 6

Threshold effect analysis of glucose levels and 28-day mortality rate using piece-wise linear regression Outcome: 28-year mortality (With diabetes)

One-line linear regression model: $\mathrm{HR}=1.001,95 \% \mathrm{Cl}(1.000-1.002), P=0.2155$

The two-piecewise linear regression model

$\begin{array}{llll}\text { Infection point } & \text { HR } & 95 \% \mathrm{Cl} & P \text {-value } \\ <258.25 \mathrm{mg} / \mathrm{dl} & 1.00 & 1.00-1.00 & 0.8098 \\ \geq 258.25 \mathrm{mg} / \mathrm{dl} & 1.01 & 1.00-1.01 & 0.0760\end{array}$

The log-likelihood ratio test: $P=0.132$

The confidence interval of the inflection point: $98.75-260.00 \mathrm{mg} / \mathrm{dl}$

Outcome: 28-year mortality (Without diabetes)

One-line linear regression model: $\mathrm{HR}=1.004,95 \% \mathrm{Cl}(1.003-1.005), P<0.0001$

The two-piecewise linear regression model

\begin{tabular}{llll} 
Infection point & HR & $95 \% \mathrm{Cl}$ & $P$-value \\
\hline$<101.75 \mathrm{mg} / \mathrm{dl}$ & 0.98 & $0.97-0.99$ & $<0.0001$ \\
$\geq 101.75 \mathrm{mg} / \mathrm{dl}$ & 1.01 & $1.00-1.01$ & $<0.0001$
\end{tabular}

The log-likelihood ratio test: $P<0.001$

The confidence interval of the inflection point: $94.64-105.80 \mathrm{mg} / \mathrm{dl}$

Note: Adjusted by age, sex, sepsis, admission type, use of insulin, type of ICU on admission, SAPS II, MV on the first day, RRT on the first day, cardiac arrhythmias, congestive heart failure, peripheral vascular disease, valvular disease, hypertension, other neurological diseases, chronic pulmonary disease, renal failure, liver disease, AIDS, lymphoma, metastatic cancer, solid tumor, obesity, fluid and electrolyte disorders, alcohol abuse, drug abuse, and depression.

Abbreviations: $\mathrm{HR}$, hazard ratio; $\mathrm{Cl}$, confidence interval. 
Table 7

Effect of glucose levels on 28-day mortality in different ICU patients with and without diabetes Without diabetes (HR, 95\% Cl, $P$-value)

\begin{tabular}{|c|c|c|c|c|}
\hline $\begin{array}{l}\text { Type of } \\
\text { ICU }\end{array}$ & $\begin{array}{l}\geq 70,<140 \\
\mathrm{mg} / \mathrm{dl}\end{array}$ & $<70 \mathrm{mg} / \mathrm{dl}$ & $\geq 140,<200 \mathrm{mg} / \mathrm{dl}$ & $\geq 200 \mathrm{mg} / \mathrm{dl}$ \\
\hline $\mathrm{CCU}$ & 1.0 & $\begin{array}{l}2.20(0.30-16.20) \\
0.4377\end{array}$ & $\begin{array}{l}1.24(0.98-1.57) \\
0.0685\end{array}$ & $\begin{array}{l}2.27(1.58-3.27) \\
<0.0001\end{array}$ \\
\hline CSRU & 1.0 & 1.0 & $\begin{array}{l}2.56(1.73-3.77) \\
<0.0001\end{array}$ & $\begin{array}{l}3.68(1.57-8.60) \\
0.0026\end{array}$ \\
\hline MICU & 1.0 & $\begin{array}{l}1.35(0.70-2.62) \\
0.3764\end{array}$ & $\begin{array}{l}1.05(0.93-1.20) \\
0.4311\end{array}$ & $\begin{array}{l}1.43(1.16-1.76) \\
0.0008\end{array}$ \\
\hline SICU & 1.0 & $\begin{array}{l}10.17(2.49-41.57) \\
0.0012\end{array}$ & $\begin{array}{l}1.33(1.10-1.61) \\
0.0032\end{array}$ & $\begin{array}{l}2.28(1.52-3.41) \\
<0.0001\end{array}$ \\
\hline TSICU & 1.0 & $\begin{array}{l}2.59(0.34-19.43) \\
0.3559\end{array}$ & $\begin{array}{l}1.58(1.27-1.98) \\
<0.0001\end{array}$ & $\begin{array}{l}2.06(1.20-3.55) \\
0.0091\end{array}$ \\
\hline Total & 1.0 & $\begin{array}{l}1.57(0.91-2.73) \\
0.1062\end{array}$ & $\begin{array}{l}1.25(1.15-1.37) \\
<0.0001\end{array}$ & $\begin{array}{l}1.80(1.54-2.10) \\
<0.0001\end{array}$ \\
\hline
\end{tabular}

With diabetes (HR, 95\% Cl, $P$-value)

$\begin{array}{lllll}\begin{array}{l}\text { Type of } \\ \text { ICU }\end{array} & \begin{array}{l}\geq 70,<140 \\ \mathrm{mg} / \mathrm{dl}\end{array} & <70 \mathrm{mg} / \mathrm{dl} & \geq 140,<200 \mathrm{mg} / \mathrm{dl} & \geq 200 \mathrm{mg} / \mathrm{dl} \\ \text { CCU } & 1.0 & 2.95(0.39-22.51) & 1.72(1.10-2.69) & 2.35(1.44-3.81) \\ & & 0.2963 & 0.0172 & 0.0006\end{array}$

\begin{tabular}{|c|c|c|c|}
\hline CSRU & 1.0 & 1.0 & $\begin{array}{l}1.14(0.62-2.13) \\
0.6689\end{array}$ \\
\hline
\end{tabular}

\begin{tabular}{|c|c|c|c|}
\hline MICU & 1.0 & $\begin{array}{l}0.43(0.10-1.76) \\
0.2399\end{array}$ & $\begin{array}{l}0.80(0.65-0.99) \\
0.0390\end{array}$ \\
\hline
\end{tabular}

$\begin{array}{llll}\text { SICU } 1.0 & 0.00(0.00-\text { Inf) } 0.9939 & 0.97(0.66-1.42) & 1.22(0.77-1.94) \\ & & 0.8764 & 0.3956\end{array}$

\begin{tabular}{|c|c|c|c|}
\hline TSICU & 1.0 & 0.00 (0.00-Inf) 0.9980 & $\begin{array}{l}1.14(0.71-1.84) \\
0.5916\end{array}$ \\
\hline
\end{tabular}

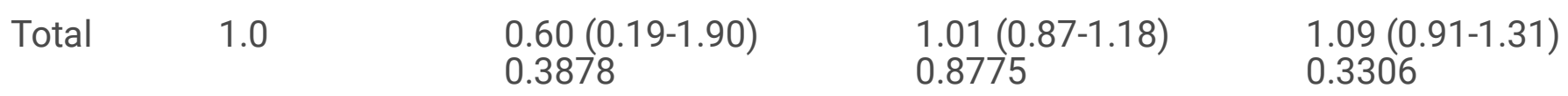

Adjusted by age, sex, sepsis, admission type, use of insulin, type of ICU on admission, SAPS II, MV on the first day, RRT on the first day, cardiac arrhythmias, congestive heart failure, peripheral vascular disease, valvular disease, hypertension, other neurological diseases, chronic pulmonary disease, renal failure, liver disease, AIDS, lymphoma, metastatic cancer, solid tumor, obesity, fluid and electrolyte disorders, alcohol abuse, drug abuse, and depression.

Abbreviations: $\mathrm{HR}$, hazard ratio; $\mathrm{Cl}$, confidence interval; ICU: intensive care unit; SAPS II: simplified acute physiology score II; CCU: coronary care unit; CSRU: cardiac surgery recovery unit; MICU: medical intensive care unit; SICU: surgical intensive care unit; TSICU: trauma/surgical intensive care unit. 


\section{Discussion}

In this study, we purposefully selected a well-represented study population of critically ill patients admitted to ICUs from a large critical healthcare database and investigated the clinical outcomes of these patients with and without diabetes, in addition to exploring the impact of blood glucose level at admission on the presence or absence of diabetes. We found that 1) diabetes was not a detrimental factor for critically ill patients in the ICUs, which would reduce the risk of 28-day mortality by about $29 \%$, 2) a V-shaped relationship was observed between blood glucose level and 28-day mortality in patients without diabetes, and hypoglycemia or hyperglycemia should be avoided, especially in patients admitted to the SICU, CSRU, and CCU; for patients with diabetes, no optimal threshold for glucose has been identified, and an elevated blood glucose level does not appear to be associated with a poor prognosis and is perhaps beneficial for certain ICU patients, and 3) particular attention should be paid to hypoglycemic events in critically ill patients without diabetes in the SICU and hyperglycemic events in all critically ill patients in the CCU and CSRU regardless of the presence of diabetes, which warrants the attention of clinicians.

Currently, the mechanisms underlying what appears to be a predominantly neutrally or protective link between diabetes and mortality in critically ill patients continue to be elusive. If diabetes is associated with decreased mortality in patients with critical illness, just a single mechanism may not be involved. Biologically, part of the potential mechanism may be that glucose plays a critical role in the function of activated immune cells and that glucose is a key contributor to energy production and maintenance of immune cell functions as well as the synthesis of immunomodulators [31-33]. Furthermore, diabetic patients develop a tolerance to hyperglycemia because of chronically elevated blood glucose concentrations, making the harmful hyperglycemia transform into an "energy factory" [34], and for the harmful effects to persist, higher blood glucose concentrations would be required [35, 36]. Namely, the smoothing splines revealed graphically that blood glucose level has a relatively small effect on patients with diabetes, and in subsequent further analyses, elevated blood glucose was not statistically associated with an increase in 28-day mortality. Our results also revealed an association between the use of insulin and diabetes on 28-day mortality, that is, there was a significant reduction in 28-day mortality in patients with diabetes who used insulin, whereas no such difference between patients with and without diabetes who did not use insulin was observed. Further analysis of the with or without insulin used cohort revealed that only 1732 patients $(11.85 \%)$ had diabetes in the non-insulin cohort, which may have weakened the decreasing effect of 28-day mortality in the diabetes cohort and resulted in the negative findings. However, this does not completely refute the association between diabetes and favorable outcomes, from an HR of 0.86 in multivariable analysis to an HR of 0.76 in stratified analysis, suggesting that the insulin use further reduced 28-day mortality in diabetic patients. The underlying mechanisms have been partially explained in previous studies with dysfunctional autophagy in critically ill patients, 
which plays a key role in both host defense and cell survival [37, 38]; insulin not only plays a role in glucose regulation but also inhibits the autophagic catabolic process [39]. Still, the occurrence of hypoglycemia resulting from the use of insulin should not be ignored. Although the incidence of hypoglycemia was high in the two Leuven studies [4,5], the condition of patients who experienced hypoglycemia did not worsen when compared to that in who did not experience hypoglycemia. The use of intensive insulin therapy to lower the blood glucose level to normal values requires careful monitoring of blood glucose, as classical neurological symptoms can be offset by sedation or underlying mental status disorders.

In terms of glucose concentrations, glycemic control in critically ill patients is still an area of considerable concern. The initial recognition of the potentially detrimental effects of hyperglycemia prompted a sequence of studies that targeted intensive insulin treatment strategies with the goal of tight glycemic control. With the accumulation of knowledge, however, there have been mixed results regarding interventions for intensive insulin therapy, i.e., the excessive pursuit of tight glycemic control in critically ill patients is exactly a counterproductive step $[4-7,14-19,40-44]$. On the basis of the data from the two Leuven studies, it was considered practical to achieve blood glucose levels of $80-110 \mathrm{mg} / \mathrm{dl}$ (rather than $180-200 \mathrm{mg} / \mathrm{dl}$ ) [4, 5]. The NICE-SUGAR study concluded that a blood glucose target of $180 \mathrm{mg} / \mathrm{dl}$ or less was less likely to result in mortality than a target of 81-108 mg/dl [7]. Krinsley et al. adopted different glycemic control strategies on the basis of diabetes status and hemoglobin A1c (HbA1c) levels in critically ill patients and found that a blood glucose level of 80-140 mg/dl was safe and effective in patients without diabetes and in those with diabetes but with a low HbA1c level; however, for patients with diabetes and $\mathrm{HbA} 1 \mathrm{c}$ levels greater than $7 \%$, the glycemic target remains ambiguous [19]. These findings mean that the moderate glycemic control strategy has been widely established in critically ill patients. Additionally, the quality of glycemic control can have an impact on clinical outcomes [20,45]. Glycemic variability has been found to be an independent risk factor for adverse outcomes in critically ill patients $[15,46-48]$. Uyttendaele et al. found that the quality of glycemic control in critically ill patients is related prognostically rather than because of the metabolic status [20]. However, the study conducted by Krinsley et al. suggested that diabetes status modulates glycemic control and mortality, as shown by the fact that diabetic patients may benefit from a higher glycemic target range compared to critically ill patients without diabetes $[15,48]$. In our opinion, diabetes affects organisms in many aspects, not only resulting in abnormal blood glucose levels but also in the inflammatory response to the disease itself and to factors such as trauma, which can disrupt insulin sensitivity. However, the quality of glycemic control also affects blood glucose levels, with hypoglycemia, hyperglycemia, and glucose variability having deleterious effects on clinical outcomes. Currently, an accurate answer regarding optimal blood glucose concentrations remains elusive. Moreover, the complexity and variability of conducting large samples of clinical trials is well known. Our findings were consistent with the "personalized" treatment strategy for patients with and without diabetes. In our study, for patients without diabetes, the glucose concentration corresponding to the lowest 28-day mortality was $101.75 \mathrm{mg} / \mathrm{dl}(95 \% \mathrm{Cl} 94.64-105.80 \mathrm{mg} / \mathrm{dl}$ ), whereas for critically ill patients with diabetes, hyperglycemia did not significantly increase the 28-day mortality, and they even benefited from a higher blood glucose level (up to $200 \mathrm{mg} / \mathrm{dl}$ ), with the exception of 
patients admitted to the CCU and CSRU. Our study primarily established an optimal threshold for the glycemic range by retrospective analysis of a large sample, which may potentially inform the practice of glycemic control and treatment strategies in critically ill patients. Nevertheless, it should be noted that we used blood glucose concentrations at ICU admission, and we acknowledged that this might not be fully extrapolated to the optimal range of glycemic control.

There are limitations of our study as well. First, we attempted to obtain information on the plasma glucose levels at ICU admission to eliminate the influence of interventions on glucose levels, but we were unable to definitively state whether the interventions that the patients received before ICU admission, such as intravenous fluid administration and steroid hormone injection, affected glucose levels. Second, as no data on HbA1c levels are available yet, we cannot exclude the possibility of new-onset diabetes. Indeed, in previous studies on measuring $\mathrm{HbA} 1 \mathrm{c}$ in patients without diabetes, it was found that $5.5 \%$, $6.8 \%$, and $9.3 \%$ of critically ill patients had higher than normal HbA1c levels [49-51], confirming that certain patients may have had undetected diabetes before ICU admission. In addition, it should be emphasized that our study did not differentiate the patients' diabetes type (e.g., type 1 or type 2). Third, we were unable to obtain information on the duration, severity, and complications of diabetes, as well as medication prescribed and therefore could not measure the impact of these factors on the outcomes. We used different models, including inverse-probability-weighted analysis, to investigate the independent role of diabetes and the clinical outcomes, but as with all retrospective studies, it was possible that residual confounders may exist. However, these clinical and electronic data were prospectively collected and independently measured, which makes them not easily amenable to manipulation. In addition, with the calculation of E-value to quantify the potential impact of unmeasured confounders, we found that unmeasured confounders are not likely to contribute to the overall effect. Fourth, we should be cautious in interpreting these results, as the results of a correlation analysis should not be mistaken for proof of causality. Finally, as the single-center study design results in reduced external validity, the aspects of glycemic control strategies and mortality reduction differing between critically ill patients with and without diabetes warrant prospective studies that can address the aforementioned limitations.

\section{Conclusions}

From this retrospective review of the prospectively collected data, the non-detrimental effect of diabetes on the short-term prognosis of critically ill patients was further confirmed, which would reduce 28-day mortality by approximately $29 \%$. Furthermore, for non-diabetic patients, the glucose level corresponding to the lowest 28-day mortality was $101.75 \mathrm{mg} / \mathrm{dl}(95 \% \mathrm{Cl} 94.64-105.80 \mathrm{mg} / \mathrm{dl})$, and hypoglycemic or hyperglycemic events should be avoided as much as possible, especially in patients admitted to the SICU, CSRU, and CCU. Moreover, for diabetic patients other than those admitted in the CCU and CSRU, elevated blood glucose levels do not appear to be associated with a poor prognosis and could benefit the patients as well. Finally, clinicians should be particularly attentive to hypoglycemic events in critically ill patients without diabetes in the SICU and to hyperglycemic events in critically ill patients in the CCU and CSRU, regardless of the presence of diabetes. 


\section{Abbreviations}

ICU: intensive care unit; SAPS II: simplified acute physiology score II; AIDS: acquired immune deficiency syndrome; PSM: propensity score matching; SQL: structure query language; IQR: interquartile range; $\mathrm{Cl}$, confidence interval; HR: hazard ratio; CCU: coronary care unit; CSRU: cardiac surgery recovery unit; MICU: medical intensive care unit; SICU: surgical intensive care unit; TSICU: trauma/surgical intensive care unit.

\section{Declarations}

\section{Ethics approval and consent to participate:}

The institutional review boards of Beth Israel Deaconess Medical Center and Massachusetts Institute of Technology Affiliates have approved access to the database (Record ID: 33460949).

\section{Consent for publication:}

Not applicable.

\section{Availability of data and materials:}

The raw data itself is from a third-party dataset available from MIMIC-III, a freely accessible critical care database. Reproduction of their data is not permitted according to the Data Use Agreement of the database but access can be requested here: https://mimic.physionet.org/gettingstarted/access.

\section{Competing interests:}

The all authors claim no conflicts of interests.

\section{Funding:}

This work was supported by the National Natural Science Foundation of China [Grant number: 81670066]; the Major Science and Technology Planning Project of Guangdong Province, China [Grant number: 2016A020216009]; the Critical Care Research Funding of the Aesculap Academy (2017); and the Guangdong Basic and Applied Basic Research Foundation, China [Grant number: 2019A1515011198].

\section{Author contributions:}

Shan Lin designed the study and wrote the draft of this manuscript, Shan Lin and Shanhui Ge mainly performed data extraction and statistical analysis, Wanmei He prepared the tables and figures, Mian 


\section{Acknowledgments:}

Thanks to Ruizhe Wang (Tencent Technology Co., Ltd. Shenzhen, Guangdong, China ) for his help in the SQL code.

\section{References}

1. Centers for Disease Control and Prevention. National Diabetes Statistics Report, 2020. Atlanta, GA: Centers for Disease Control and Prevention, US Department of Health and Human Services; 2020.

2. Muller L, Gorter K J, Hak E, et al. Increased risk of common infections in patients with type 1 and type 2 diabetes mellitus. Clin Infect Dis. 2005;41(3):281-288.

3. Schuetz P, Castro P, Shapiro N I. Diabetes and sepsis: preclinical findings and clinical relevance. Diabetes care. 2011;34(3):771-778.

4. Van Den Berghe G, Wouters P, Weekers F, et al. Intensive insulin therapy in critically ill patients. N Engl J Med. 2001;345(19):1359-1367.

5. Van den Berghe G, Wilmer A, Hermans G, et al. Intensive insulin therapy in the medical ICU. N Engl J Med. 2006;354(5): 449-461.

6. Brunkhorst F M, Engel C, Bloos F, et al. Intensive insulin therapy and pentastarch resuscitation in severe sepsis. N Engl J Med. 2008;358(2):125-139.

7. Nice-Sugar Study Investigators. Intensive versus conventional glucose control in critically ill patients. N Engl J Med. 2009;360(13): 1283-1297.

8. Michalia M, Kompoti M, Koutsikou A, et al. Diabetes mellitus is an independent risk factor for ICUacquired bloodstream infections. Intensive Care Med. 2009;35(3):448-454.

9. Abdelhamid Y A, Plummer M P, Finnis M E, et al. Long-term mortality of critically ill patients with diabetes who survive admission to the intensive care unit. Crit Care Resusc. 2017;19(4):303.

10. Christiansen CF, Johansen MB, Christensen S, O'Brien JM, Tønnesen E, Sørensen HT. Type 2 diabetes and 1-year mortality in intensive care unit patients. Eur J Clin Invest. 2013;43(3):238-247.

11. Vincent JL, Preiser JC, Sprung CL, Moreno R, Sakr Y. Insulin-treated diabetes is not associated with increased mortality in critically ill patients. Crit Care. 2010;14(1):R12.

12. Graham B B, Keniston A, Gajic 0 , et al. Diabetes mellitus does not adversely affect outcomes from a critical illness. Crit care med. 2010;38(1):16-24.

13. Siegelaar S E, Hickmann M, Hoekstra J B L, et al. The effect of diabetes on mortality in critically ill patients: a systematic review and meta-analysis. Crit Care. 2011;15(5):1-12.

14. Sechterberger M K, Bosman R J, Oudemans-van Straaten H M, et al. The effect of diabetes mellitus on the association between measures of glycaemiccontrol and ICU mortality: a retrospective cohort study. Crit Care. 2013;17(2):R52. 
15. Krinsley J S, Egi M, Kiss A, et al. Diabetic status and the relation of the three domains of glycemic control tomortality in critically ill patients: an international multicenter cohort study. Crit Care. 2013;17(2):R37.

16. Yatabe T, Inoue S, Sakaguchi M, Egi M. The optimal target for acute glycemic control in critically ill patients: a network meta-analysis. Intensive Care Med. 2017;43(1):16-28.

17. Lanspa MJ, Hirshberg EL, Phillips GD, Holmen J, Stoddard G, Orme J. Moderate glucose control is associated with increased mortality compared with tight glucose control in critically ill patients without diabetes. Chest. 2013;143(5):1226-1234.

18. Ling Y, Li X, Gao X. Intensive versus conventional glucose control in critically ill patients: a metaanalysis of randomized controlled trials. Eur J Intern Med. 2012;23(6):564-574.

19. Krinsley JS, Preiser JC, Hirsch IB. SAFETY AND EFFICACY OF PERSONALIZED GLYCEMIC CONTROL IN CRITICALLY ILL PATIENTS: A 2-YEAR BEFORE AND AFTER INTERVENTIONAL TRIAL. Endocr Pract. 2017;23(3):318-330.

20. Uyttendaele V, Dickson JL, Shaw GM, Desaive T, Chase JG. Untangling glycaemia and mortality in critical care. Crit Care. 2017;21(1):152.

21. Johnson AE, Pollard TJ, Shen L, et al. MIMIC-III, a freely accessible critical care database. Sci Data. 2016;3:160035.

22. Le Gall JR, Lemeshow S, Saulnier F. A new Simplified Acute Physiology Score (SAPS II) based on a European/North American multicenter study. JAMA. 1993;270(24):2957-2963.

23. Johnson A, Stone DJ, Celi LA, Pollard TJ. The MIMIC code repository: enabling reproducibility in critical care research. J Am Med Inform Assoc. 2017;25(1):32-39.

24. Lin S, Ge S, He W, Zeng M. Association of delayed time in the emergency department with the clinical outcomes for critically ill patients. Q J Med. DOI: 10.1093/qjmed/hcaa192.

25. Rubin D B. Using propensity scores to help design observational studies: application to the tobacco litigation. Health Serv Outcomes Res Methodol. 2001;2:169-188.

26. Robins JM, Hernán MA, Brumback B. Marginal structural models and causal inference in epidemiology. Epidemiology. 2000;11(5):550-560.

27. Austin P C. Using the standardized difference to compare the prevalence of a binary variable between two groups in observational research. Commun Stat Simul Comput. 2009;38(6): 1228-1234.

28. VanderWeele TJ, Ding P. Sensitivity Analysis in Observational Research: Introducing the E-Value. Ann Intern Med. 2017;167(4):268-274.

29. Haneuse S, VanderWeele TJ, Arterburn D. Using the E-Value to Assess the Potential Effect of Unmeasured Confounding in Observational Studies. JAMA. 2019;321(6):602-603.

30. DiCiccio T J, Efron B. Bootstrap confidence intervals. Stat Sci. 1996:189-212.

31. Ganeshan K, Chawla A. Metabolic regulation of immune responses. Annu Rev Immunol. 2014;32:609-634. 
32. Pearce EL, Pearce EJ. Metabolic pathways in immune cell activation and quiescence. Immunity. 2013;38(4):633-643.

33. Loftus RM, Finlay DK. Immunometabolism: Cellular Metabolism Turns Immune Regulator. J Biol Chem. 2016;291(1):1-10.

34. Van Niekerk G, Davis T, Engelbrecht A M. Hyperglycaemia in critically ill patients: the immune system's sweet tooth. Crit Care. 2017;21(1):202.

35. Egi M, Bellomo R, Stachowski E, et al. Blood glucose concentration and outcome of critical illness: the impact of diabetes. Crit Care Med. 2008;36(8):2249-55.

36. Falciglia M, Freyberg RW, Almenoff PL, D’Alessio DA, Render ML. Hyperglycemia-related mortality in critically ill patients varies with admission diagnosis. Crit Care Med. 2009;37(12):3001-3009.

37. van Niekerk G, Loos B, Nell T, Engelbrecht AM. Autophagy-A free meal in sickness-associated anorexia. Autophagy. 2016;12(4):727-734.

38. Vanhorebeek I, Gunst J, Derde S, et al. Insufficient activation of autophagy allows cellular damage to accumulate in critically ill patients. J Clin Endocrinol Metab. 2011;96(4):E633-E645.

39. Pfeifer U. Inhibition by insulin of the physiological autophagic breakdown of cell organelles. Acta Biol Med Ger. 1977;36(11-12):1691-1694.

40. Arabi Y M, Dabbagh O C, Tamim H M, et al. Intensive versus conventional insulin therapy: a randomized controlled trial in medical and surgical critically ill patients. Critical care med. 2008;36(12):3190-3197.

41. Van den Berghe G, Wilmer A, Milants I, et al. Intensive insulin therapy in mixed medical/surgical intensive care units: benefit versus harm. Diabetes. 2006;55(11):3151-3159.

42. Finney S J, Zekveld C, Elia A, et al. Glucose control and mortality in critically ill patients. Jama. 2003;290(15):2041-2047.

43. Preiser J C, Devos P, Ruiz-Santana S, et al. A prospective randomised multi-centre controlled trial on tight glucose control by intensive insulin therapy in adult intensive care units: the Glucontrol study. Intensive care med. 2009;35(10):1738.

44. De La Rosa G D C, Donado J H, Restrepo A H, et al. Strict glycaemic control in patients hospitalised in a mixed medical and surgical intensive care unit: a randomised clinical trial. Crit care, 2008;12(5):R120.

45. Rodbard D. Interpretation of continuous glucose monitoring data: glycemic variability and quality of glycemic control. Diabetes Technol Ther. 2009;11 Suppl 1:S55-S67.

46. Lanspa MJ, Dickerson J, Morris AH, Orme JF, Holmen J, Hirshberg EL. Coefficient of glucose variation is independently associated with mortality in critically ill patients receiving intravenous insulin. Crit Care. 2014;18(2):R86.

47. Krinsley JS. Glycemic variability: a strong independent predictor of mortality in critically ill patients. Crit Care Med. 2008;36(11):3008-3013. 
48. Krinsley JS, Preiser JC. Time in blood glucose range 70 to $140 \mathrm{mg} / \mathrm{dl}>80 \%$ is strongly associated with increased survival in non-diabetic critically ill adults. Crit Care. 2015;19(1):179.

49. Plummer MP, Bellomo R, Cousins $C E$, et al. Dysglycaemia in the critically ill and the interaction of chronic and acute glycaemia with mortality. Intensive Care Med. 2014;40(7):973-980.

50. van Vught LA, Wiewel MA, Klein Klouwenberg PM, et al. Admission Hyperglycemia in Critically III Sepsis Patients: Association With Outcome and Host Response. Crit Care Med. 2016;44(7):13381346.

51. Carpenter DL, Gregg SR, Xu K, Buchman TG, Coopersmith CM. Prevalence and Impact of Unknown Diabetes in the ICU. Crit Care Med. 2015;43(12):e541-e550.

\section{Figures}




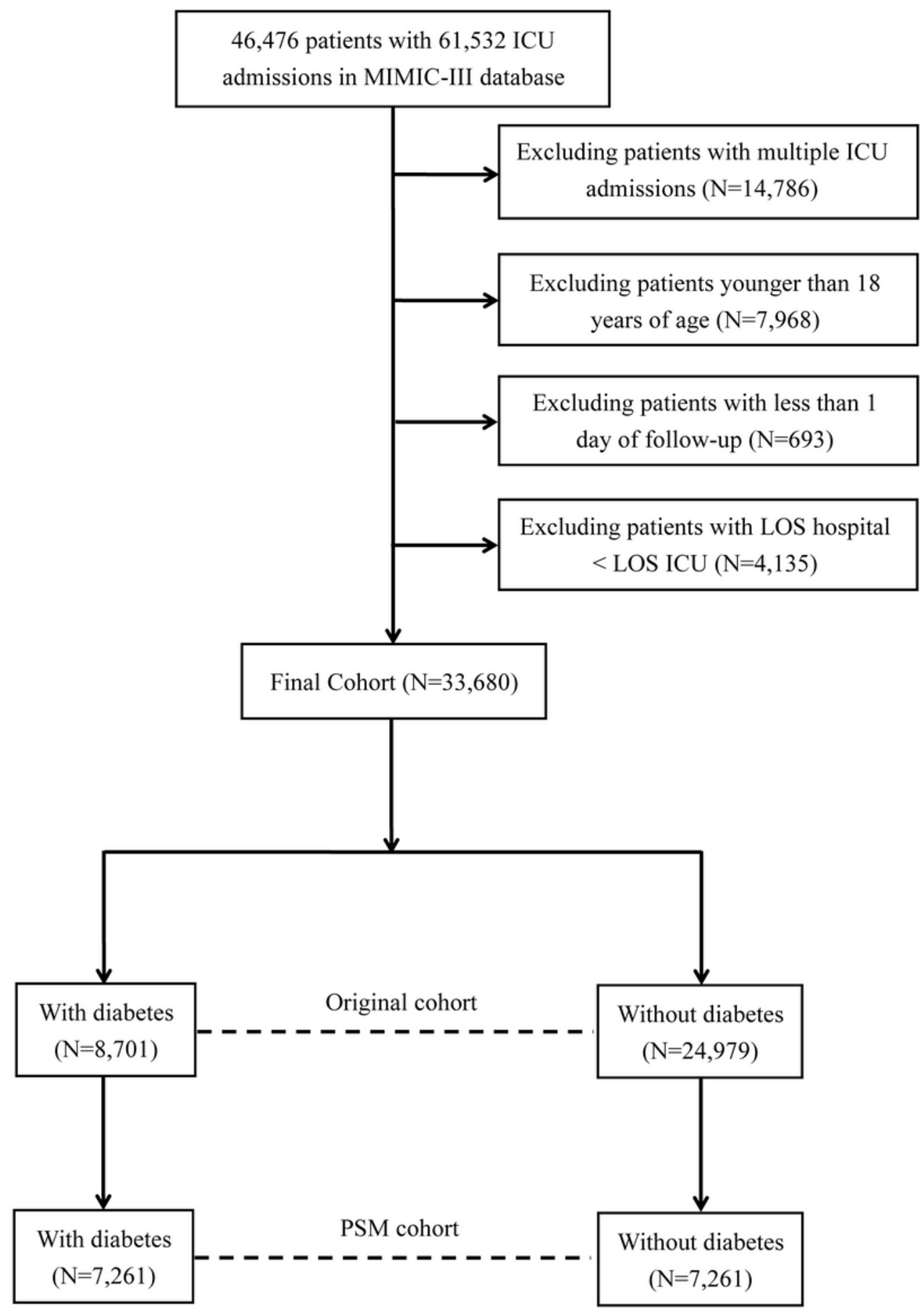

\section{Figure 1}

Flow chart of study participants Abbreviations: ICU: intensive care unit; LOS: length of stay; PSM: propensity score matching. 


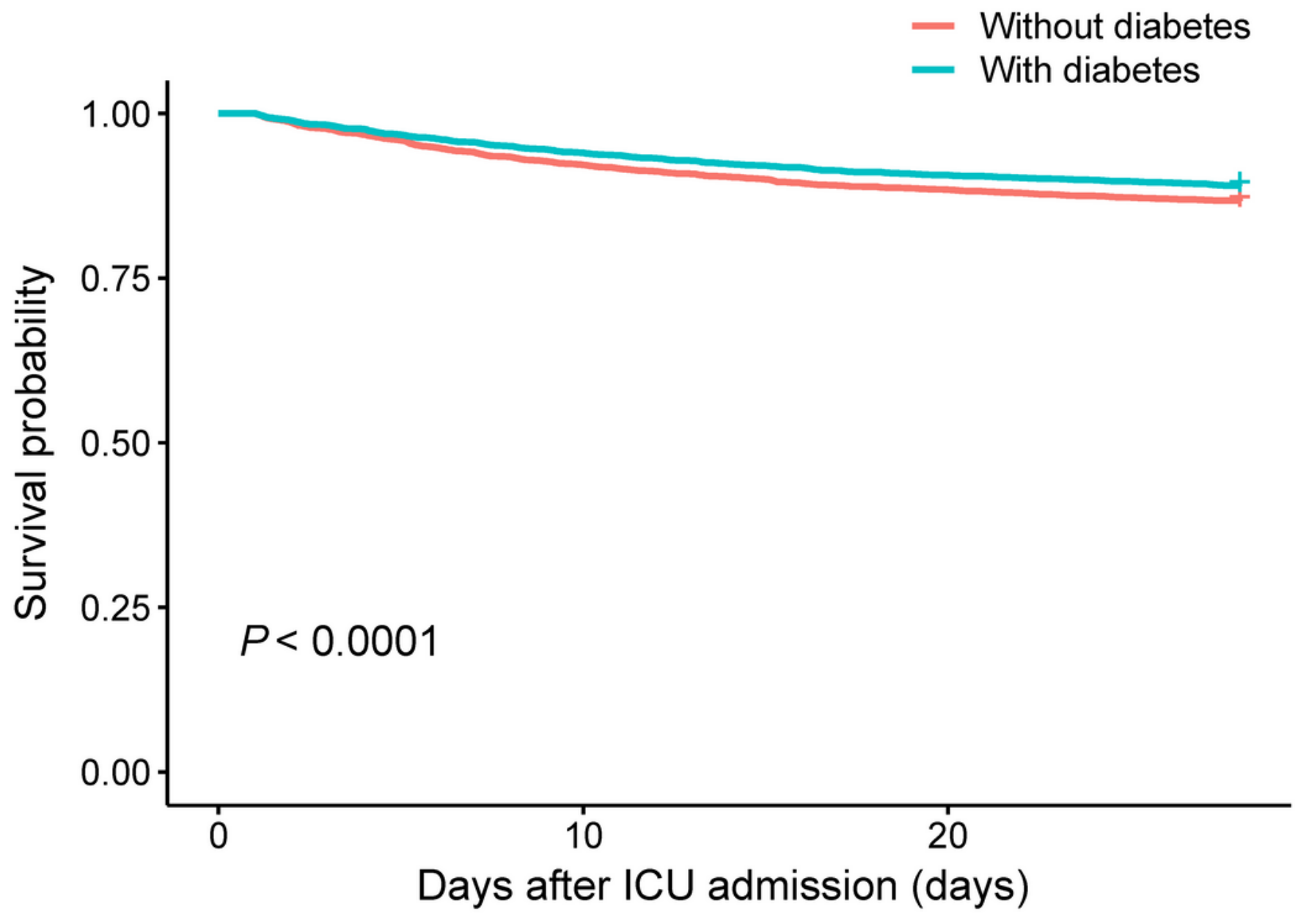

Number at risk

\begin{tabular}{|ccc}
7261 & 6692 & 6419 \\
7261 & 6827 & 6581 \\
\hline 0 & & 10 \\
& Days after ICU admission (days)
\end{tabular}

Figure 2

Kaplan-Meier survival curve of the PSM cohort Abbreviations: ICU: intensive care unit; PSM: propensity score matching. 


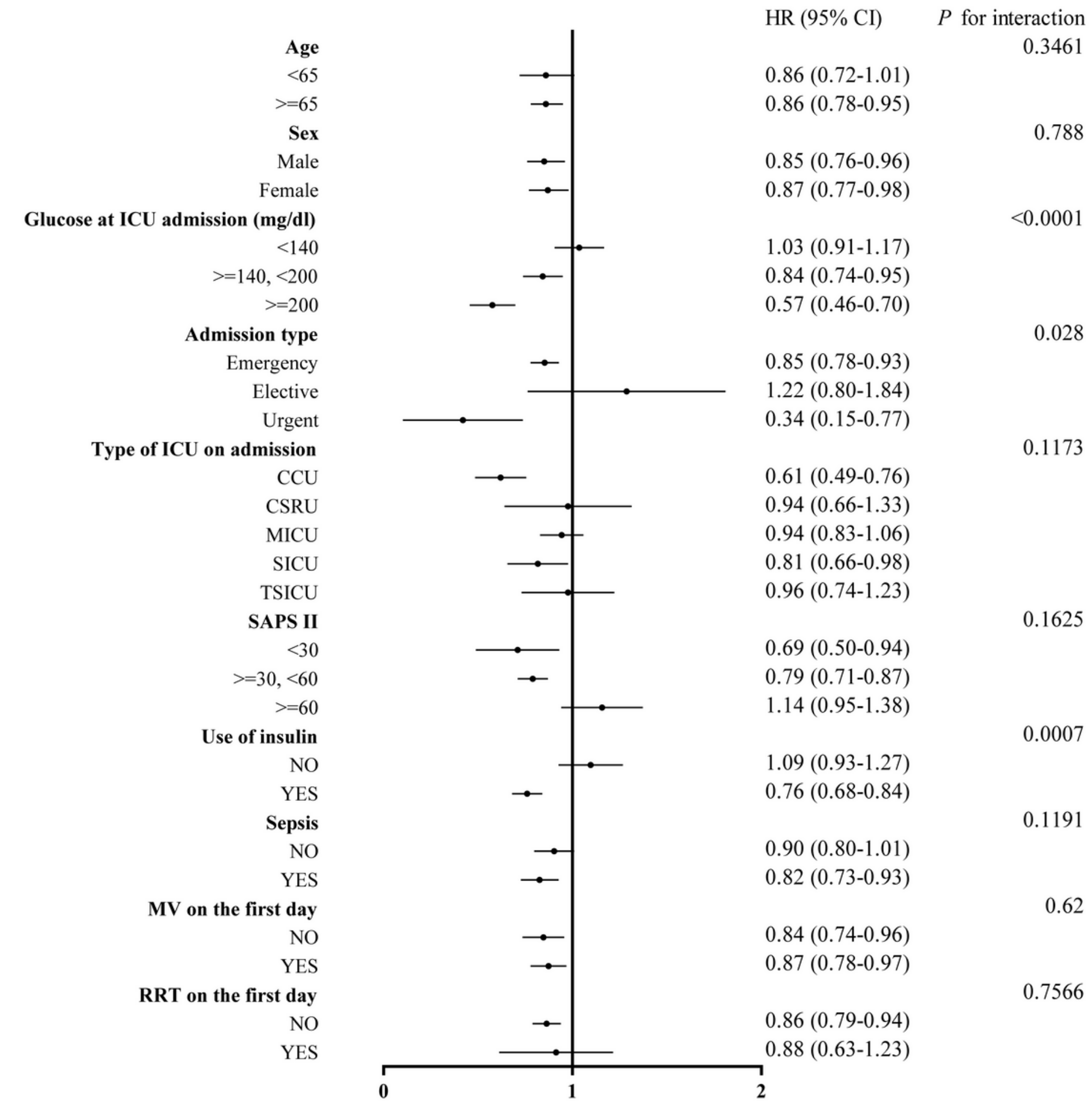

\section{Figure 3}

Forest plot of diabetes on 28-day mortality in prespecified and exploratory subgroups in each subgroup Abbreviations: ICU: intensive care unit; SAPS II: simplified acute physiology score II; CCU: coronary care unit; CSRU: cardiac surgery recovery unit; MICU: medical intensive care unit; SICU: surgical intensive care unit; TSICU: trauma/surgical intensive care unit; MV: mechanical ventilation; RRT: renal replacement therapy. 


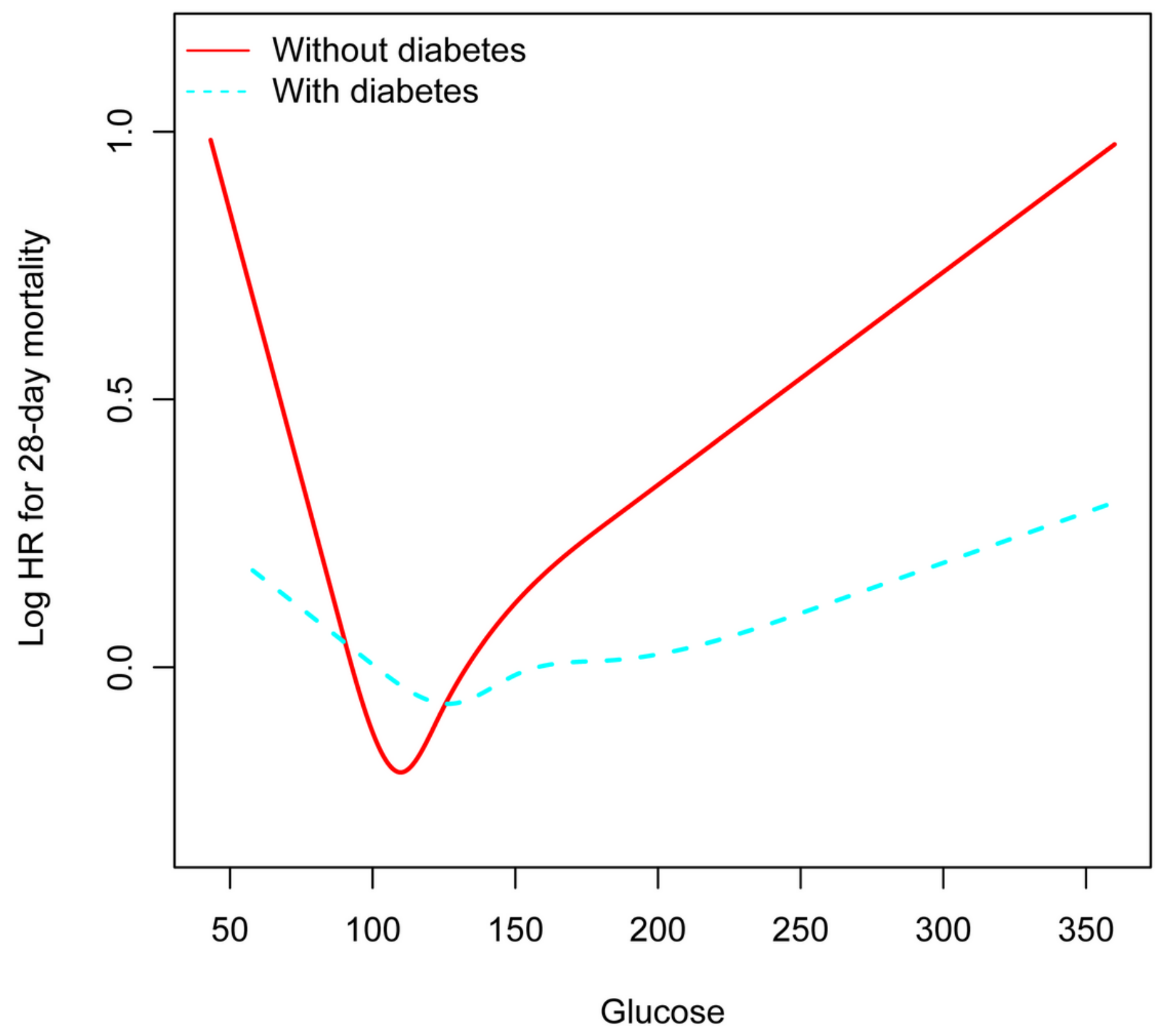

Figure 4

Association between glucose and 28-day mortality in critically ill patients with and without diabetes Abbreviations: HR: hazard ratio. 


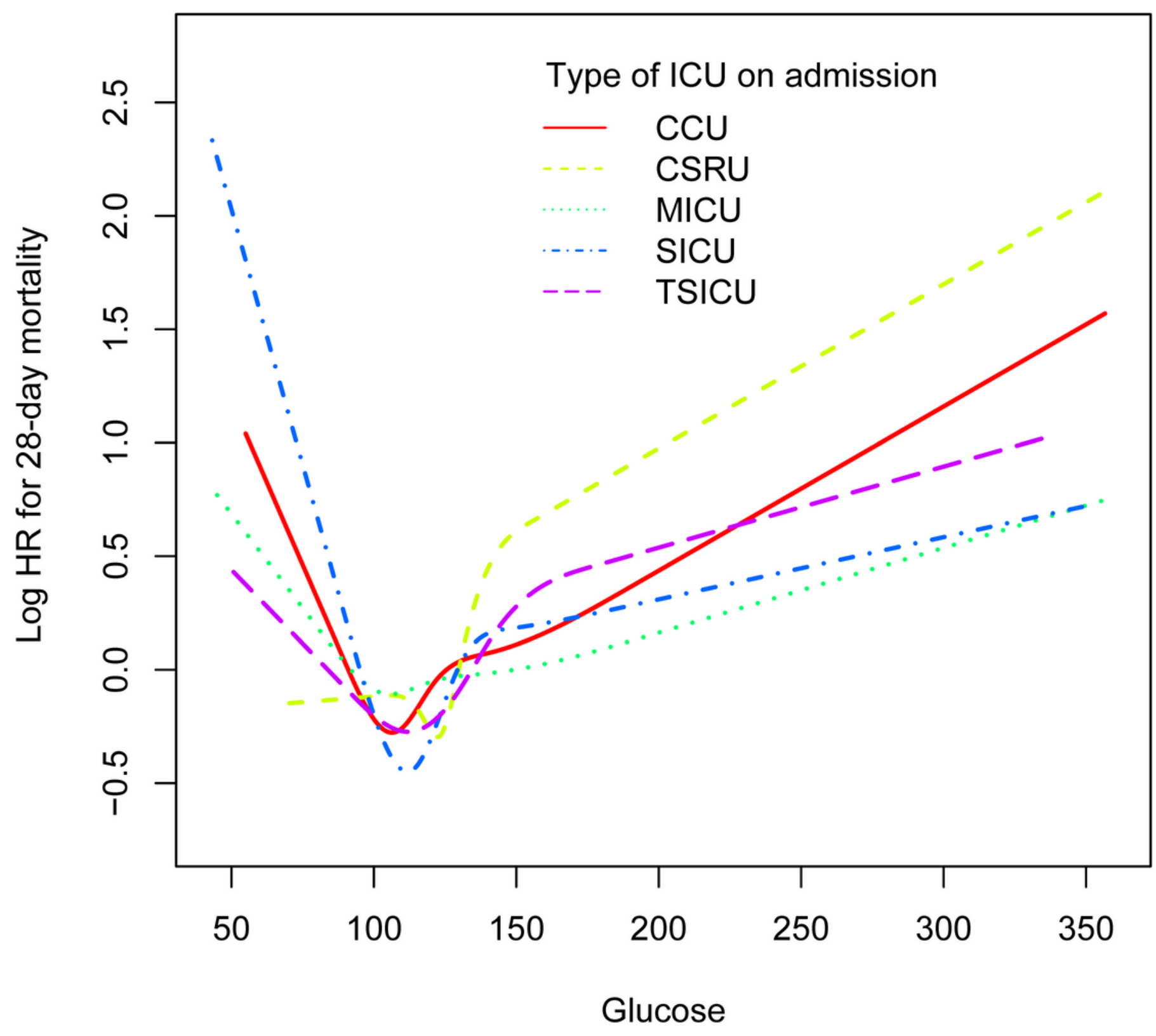

Figure 5

Association between glucose and 28-day mortality in critically ill patients without diabetes admitted to different ICU types Abbreviations: HR: hazard ratio; ICU: intensive care unit; CCU: coronary care unit; CSRU: cardiac surgery recovery unit; MICU: medical intensive care unit; SICU: surgical intensive care unit; TSICU: trauma/surgical intensive care unit. 


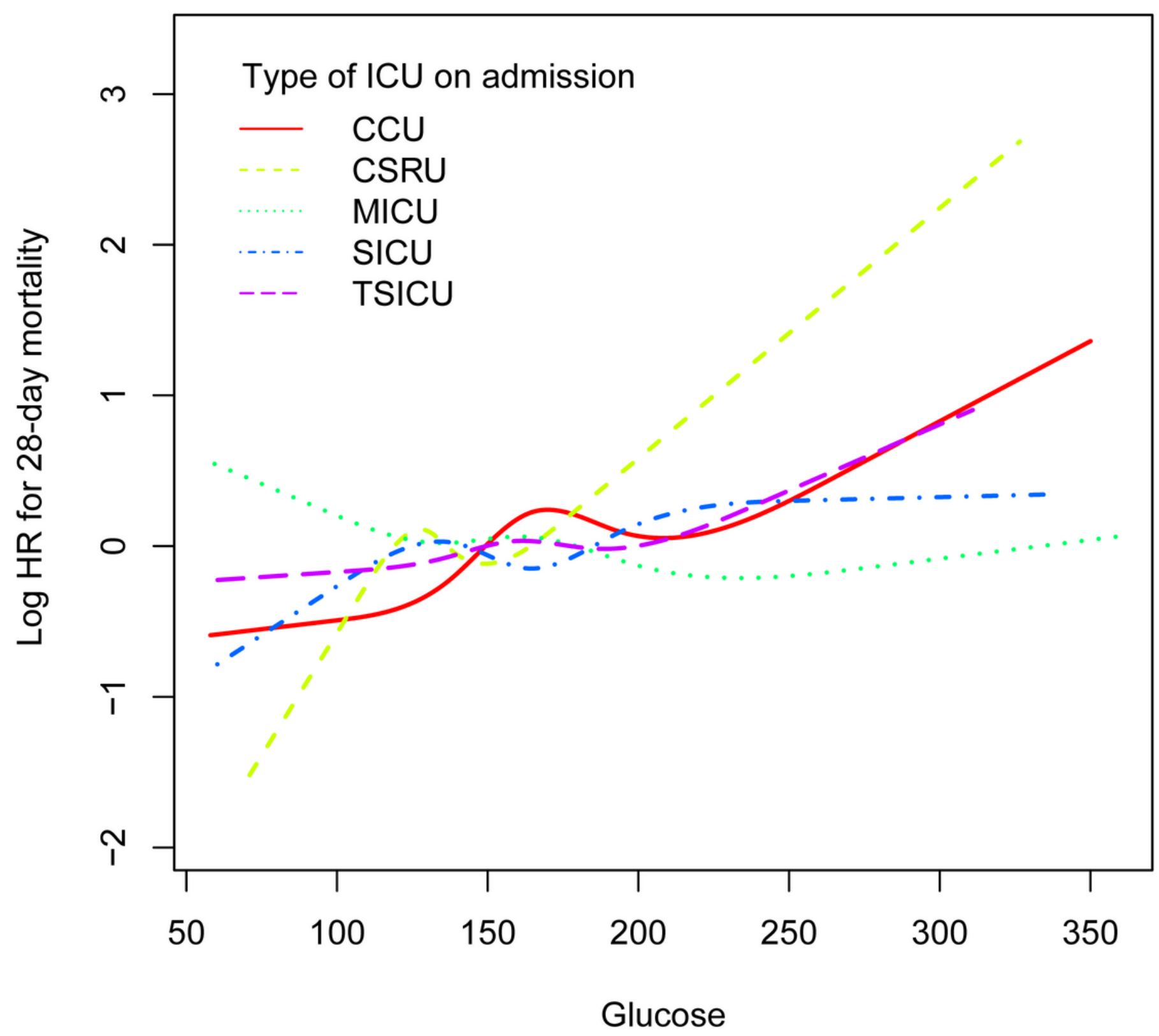

Figure 6

Association between glucose and 28-day mortality in critically ill patients with diabetes admitted to different ICU types Abbreviations: HR: hazard ratio; ICU: intensive care unit; CCU: coronary care unit; CSRU: cardiac surgery recovery unit; MICU: medical intensive care unit; SICU: surgical intensive care unit; TSICU: trauma/surgical intensive care unit. 